\title{
NON-ARCHIMEDEAN MODELS OF MORPHOGENESIS
}

\author{
W. A. ZÚÑIGA-GALINDO
}

\begin{abstract}
We study a $p$-adic reaction-diffusion system and the associated Turing patterns. We establish an instability criteria and show that the Turing patterns are not classical patterns consisting of alternating domains. Instead of this, a Turing pattern consists of several domains (clusters), each of them supporting a different pattern but with the same parameter values. This type of patterns are typically produced by reaction-diffusion equations on large networks.
\end{abstract}

\section{INTRODUCTION}

In 1952, A. Turing proposed that under certain conditions chemicals can react and diffuse in such a way as to produce steady state heterogeneous spatial patterns of chemical (or morphogen) concentration. In the case of two morphogens interacting, the model proposed by Turing has the form:

$$
\left\{\begin{array}{l}
\frac{\partial u}{\partial t}(x, t)=\gamma f(u, v)+\frac{\partial^{2} u}{\partial x^{2}}(x, t) \\
\frac{\partial v}{\partial t}(x, t)=\gamma g(u, v)+d \frac{\partial^{2} v}{\partial x^{2}}(x, t),
\end{array}\right.
$$

with suitable boundary conditions. In this model $u, v$ represent the concentrations of the two morphogens, $f$ and $g$ represent the reaction kinetics, and $d$ is the ratio of diffusion, and $\gamma$ represents the relative strength of the reaction terms.

Typically $u(x, t)$ and $v(x, t)$ are interpreted to be the local densities of the activator and inhibitor species. Functions $f(u, v)$ and $g(u, v)$ specify the local dynamics of the activator, which autocatalytically enhances its own production, and of the inhibitor, which suppresses the activator growth. The Turing instability occurs when the parameter $d$ exceeds a threshold $d_{c}, 22$, , 15. This event drives to a spontaneous development of a spatial pattern formed by alternating activator-rich and activator-poor patches. Turing instability in activator-inhibitor systems establishes a paradigm of non-equilibrium self-organization, which has been extensively studied for biological and chemical processes.

In the 70s, Othmer and Scriven started the study of the Turing instability in network-organized systems [17-[18. Since then, reaction-diffusion models on networks have been studied intensively, see e.g. [2], [5], [6], 9], [12], 14], [16, [17, 18, 20, 23, 25, 26, 28, and the references therein.

In the discrete case, the continuous media is replaced by a network (an unoriented graph $\mathcal{G}$, which plays the role of discrete media). The analog of operator $\Delta$ is the

2020 Mathematics Subject Classification. Primary: 35K57, 47S10. Secondary: 92C42.

Key words and phrases. Reaction-diffusion equations, Turing patterns, $p$-adic analysis.

The author was partially supported by Conacyt Grant No. 217367 (Mexico), and by the Debnath Endowed Professorship (UTRGV, USA). 
Laplacian of the graph $\mathcal{G}$, which is defined as

$$
\left[L_{J I}\right]_{J, I \in V(\mathcal{G})}=\left[A_{J I}-\gamma_{I} \delta_{J I}\right]_{J, I \in V(\mathcal{G})},
$$

where $\left[A_{J I}\right]_{J, I \in V(\mathcal{G})}$ is the adjacency matrix of $\mathcal{G}$ and $\gamma_{I}$ is the degree of $I$. The network analogue of (1.1) is

$$
\left\{\begin{array}{l}
\frac{\partial u_{J}}{\partial t}=f\left(u_{J}, v_{J}\right)+\varepsilon \sum_{I} L_{J I} u_{I} \\
\frac{\partial v_{J}}{\partial t}=g\left(u_{J}, v_{J}\right)+\varepsilon d \sum_{I} L_{J I} v_{I}
\end{array}\right.
$$

In the last fifty years, Turing patterns produced by reaction-diffusion systems on networks have been studied intensively, see e.g. [5, [9, 12, [16, 17, [18, 26, [28 and the references therein. Nowadays, there is a large amount of experimental results about the behavior of these systems, obtained mainly via computer simulations using large random networks. The investigations of the Turing patterns for large random networks have revealed that, whereas the Turing criteria remain essentially the same, as in the classical case, the properties of the emergent patterns are very different. In [16, by using a physical argument, Nakao and Mikhailov establish that Turing patterns with alternating domains cannot exist in the network case, and only several domains (clusters) occur. Multistability, that is, coexistence of a number of different patterns with the same parameter values, is typically found and hysteresis phenomena are observed. They used mean-field approximation to understand the Turing patterns when $d>d_{c}$, and proposed that the mean-field approximation is the natural framework to understand the peculiar behavior of the Turing patterns on networks. This program was carried out in 28, using $p$-adic analysis.

In [28] we established that the $p$-adic reaction-diffusion system

$$
\left\{\begin{array}{l}
\frac{\partial u(x, t)}{\partial t}=f(u(x, t), v(x, t))+\varepsilon \int_{\mathcal{K}_{N}}(u(y, t)-u(x, t)) J_{N}(x, y) d y \\
\frac{\partial v(x, t)}{\partial t}=g(u(x, t), v(x, t))+\varepsilon d \int_{\mathcal{K}_{N}}(u(y, t)-u(x, t)) J_{N}(x, y) d y,
\end{array}\right.
$$

where $\mathcal{K}_{N}$ (an open compact subset) and $J_{N}(x, y)$ depends on $\left[A_{J I}\right]_{J, I \in V(\mathcal{G})}$, is a good $p$-adic continuous approximation of (1.3). The Turing instability criteria for (1.4) is essentially the same as in the classical case, but the qualitative description of the Turing patterns is the one given by Nakao and Mikhailov in terms of clustering and multistability.

In this article we study the following $p$-adic reaction-diffusion system:

$$
\left\{\begin{array}{l}
\frac{\partial u}{\partial t}(x, t)=\gamma f(u, v)-\boldsymbol{D}_{x}^{\alpha} u(x, t) ; \\
\frac{\partial v}{\partial t}(x, t)=\gamma g(u, v)-d \boldsymbol{D}_{x}^{\alpha} v(x, t),
\end{array}\right.
$$

where $x \in \mathbb{Q}_{p}, t \geq 0$, and $\boldsymbol{D}_{x}^{\alpha}$ is the Vladimirov operator. Since want to study self-organization patterns we need a 'zero flux boundary condition', which is

$$
u(x, t), v(x, t) \equiv 0 \text { for } x \in \mathbb{Q}_{p} \backslash B_{M} \text {, for any } t \geq 0,
$$

where $B_{M}$ is the ball of radius $p^{M}$ around the origin. We establish a Turing instability criteria for (1.5) and show that the Turing pattern can be described as 
a couple of convergent series involving the functions

$$
\begin{gathered}
e^{\lambda t} p^{\frac{-r}{2}} \cos \left(\left\{p^{-1} j\left(p^{r} x-n\right)\right\}_{p}\right) \Omega\left(\left|p^{r} x-n\right|_{p}\right), \\
p^{\frac{-r}{2}} \sin \left(\left\{p^{-1} j\left(p^{r} x-n\right)\right\}_{p}\right) \Omega\left(\left|p^{r} x-n\right|_{p}\right),
\end{gathered}
$$

see Theorem 1 . The Turing patterns attached to (1.5) are not classical patterns consisting of alternating domains. Instead of this, a Turing pattern consists of several domains (clusters), each of them supporting a different pattern but with the same parameter values (multistability). It is important to mention that the spectra of $\boldsymbol{D}_{x}^{\alpha}$ consists of a sequence of positive eigenvalues with infinite multiplicity, while the spectra of the operators considered in [28, Theorem 10.1] consists of a finite number of non-negative eigenvalues with finite multiplicities. In Section 5 we construct discretization of system (1.5) of type (1.3). But, In this case the matrix of the discrete is not related to adjacency matrix of a graph, see (5.1).

\section{2. $p$-Adic Analysis: Essential Ideas}

In this section we collect some basic results about $p$-adic analysis that will be used in the article. For an in-depth review of the $p$-adic analysis the reader may consult [1], 21], 24].

2.1. The field of $p$-adic numbers. Along this article $p$ will denote a prime number. The field of $p$-adic numbers $\mathbb{Q}_{p}$ is defined as the completion of the field of rational numbers $\mathbb{Q}$ with respect to the $p$-adic norm $|\cdot|_{p}$, which is defined as

$$
|x|_{p}= \begin{cases}0 & \text { if } \quad x=0 \\ p^{-\gamma} & \text { if } \quad x=p^{\gamma} \frac{a}{b},\end{cases}
$$

where $a$ and $b$ are integers coprime with $p$. The integer $\gamma:=\operatorname{ord}(x)$, with $\operatorname{ord}(0):=$ $+\infty$, is called the $p$-adic order of $x$.

Any $p$-adic number $x \neq 0$ has a unique expansion of the form

$$
x=p^{o r d}(x) \sum_{j=0}^{\infty} x_{j} p^{j},
$$

where $x_{j} \in\{0, \ldots, p-1\}$ and $x_{0} \neq 0$. By using this expansion, we define the fractional part of $x \in \mathbb{Q}_{p}$, denoted $\{x\}_{p}$, as the rational number

$$
\{x\}_{p}= \begin{cases}0 & \text { if } \quad x=0 \text { or } \operatorname{ord}(x) \geq 0 \\ p^{\operatorname{ord}(x)} \sum_{j=0}^{-\operatorname{ord}_{p}(x)-1} x_{j} p^{j} & \text { if } \quad \operatorname{ord}(x)<0 .\end{cases}
$$

In addition, any non-zero $p$-adic number can be represented uniquely as $x=$ $p^{\operatorname{ord}(x)} a c(x)$ where $a c(x)=\sum_{j=0}^{\infty} x_{j} p^{j}, x_{0} \neq 0$, is called the angular component of $x$. Notice that $|a c(x)|_{p}=1$.

For $r \in \mathbb{Z}$, denote by $B_{r}(a)=\left\{x \in \mathbb{Q}_{p} ;|x-a|_{p} \leq p^{r}\right\}$ the ball of radius $p^{r}$ with center at $a \in \mathbb{Q}_{p}$, and take $B_{r}(0):=B_{r}$. The ball $B_{0}$ equals $\mathbb{Z}_{p}$, the ring of $p$-adic integers of $\mathbb{Q}_{p}$. We also denote by $S_{r}(a)=\left\{x \in \mathbb{Q}_{p} ;|x-a|_{p}=p^{r}\right\}$ the sphere of radius $p^{r}$ with center at $a \in \mathbb{Q}_{p}$, and take $S_{r}(0):=S_{r}$. We notice that $S_{0}^{1}=\mathbb{Z}_{p}^{\times}$ (the group of units of $\mathbb{Z}_{p}$ ). The balls and spheres are both open and closed subsets in $\mathbb{Q}_{p}$. In addition, two balls in $\mathbb{Q}_{p}$ are either disjoint or one is contained in the other. 
The metric space $\left(\mathbb{Q}_{p},|\cdot|_{p}\right)$ is a complete ultrametric space. As a topological space $\left(\mathbb{Q}_{p},|\cdot|_{p}\right)$ is totally disconnected, i.e. the only connected subsets of $\mathbb{Q}_{p}$ are the empty set and the points. In addition, $\mathbb{Q}_{p}$ is homeomorphic to a Cantor-like subset of the real line, see e.g. 11, 24. A subset of $\mathbb{Q}_{p}$ is compact if and only if it is closed and bounded in $\mathbb{Q}_{p}$, see e.g. [24, Section 1.3], or [1, Section 1.8]. The balls and spheres are compact subsets. Thus $\left(\mathbb{Q}_{p},|\cdot|_{p}\right)$ is a locally compact topological space.

Notation 1. We use $\Omega\left(p^{-r}|x-a|_{p}\right)$ to denote the characteristic function of the ball $B_{r}(a)=a+p^{-r} \mathbb{Z}_{p}$. For more general sets, we use the notation $1_{A}$ for the characteristic function of a $A$.

2.2. Some function spaces. A complex-valued function $\varphi$ defined on $\mathbb{Q}_{p}$ is called locally constant if for any $x \in \mathbb{Q}_{p}$ there exist an integer $l(x) \in \mathbb{Z}$ such that

$$
\varphi\left(x+x^{\prime}\right)=\varphi(x) \text { for } x^{\prime} \in B_{l(x)} .
$$

A function $\varphi: \mathbb{Q}_{p} \rightarrow \mathbb{C}$ is called a Bruhat-Schwartz function (or a test function) if it is locally constant with compact support. In this case, we can take $l=l(\varphi)$ in (2.1) independent of $x$, the largest of such integers is called the parameter of local constancy of $\varphi$. The $\mathbb{C}$-vector space of Bruhat-Schwartz functions is denoted by $\mathcal{D}:=\mathcal{D}\left(\mathbb{Q}_{p}\right)$. We denote by $\mathcal{D}_{\mathbb{R}}:=\mathcal{D}_{\mathbb{R}}\left(\mathbb{Q}_{p}^{n}\right)$, the $\mathbb{R}$-vector space of test functions.

Since $\left(\mathbb{Q}_{p},+\right)$ is a locally compact topological group, there exists a Borel measure $d x$, called the Haar measure of $\left(\mathbb{Q}_{p},+\right)$, unique up to multiplication by a positive constant. Furthermore, $\int_{U} d x>0$ for every non-empty open set $U \subset \mathbb{Q}_{p}$, and $\int_{E+z} d x=\int_{E} d x$ for every Borel set $E \subset \mathbb{Q}_{p}$, see e.g. [7, Chapter XI]. If we normalize this measure by the condition $\int_{\mathbb{Z}_{p}} d x=1$, then $d x$ is unique. From now on we denote by $d x$ the normalized Haar measure of $\left(\mathbb{Q}_{p},+\right)$.

Given $\rho \in[0, \infty)$, we denote by $L^{\rho}:=L^{\rho}\left(\mathbb{Q}_{p}\right):=L^{\rho}\left(\mathbb{Q}_{p}, d x\right)$, the $\mathbb{C}$-vector space of all the complex valued functions $g$ satisfying $\int_{\mathbb{Q}_{p}}|g(x)|^{\rho} d x<\infty$, and $L^{\infty}$ $:=L^{\infty}\left(\mathbb{Q}_{p}\right)=L^{\infty}\left(\mathbb{Q}_{p}, d x\right)$ denotes the $\mathbb{C}$-vector space of all the complex valued functions $g$ such that the essential supremum of $|g|$ is bounded. The corresponding $\mathbb{R}$-vector spaces are denoted as $L_{\mathbb{R}}^{\rho}:=L_{\mathbb{R}}^{\rho}\left(\mathbb{Q}_{p}\right)=L_{\mathbb{R}}^{\rho}\left(\mathbb{Q}_{p}, d x\right), 1 \leq \rho \leq \infty$.

2.3. Fourier transform. Set $\chi_{p}(y)=\exp \left(2 \pi i\{y\}_{p}\right)$ for $y \in \mathbb{Q}_{p}$. The map $\chi_{p}(\cdot)$ is an additive character on $\mathbb{Q}_{p}$, i.e. a continuous map from $\left(\mathbb{Q}_{p},+\right)$ into $S$ (the unit circle considered as multiplicative group) satisfying $\chi_{p}\left(x_{0}+x_{1}\right)=\chi_{p}\left(x_{0}\right) \chi_{p}\left(x_{1}\right)$, $x_{0}, x_{1} \in \mathbb{Q}_{p}$. The additive characters of $\mathbb{Q}_{p}$ form an Abelian group which is isomorphic to $\left(\mathbb{Q}_{p},+\right)$. The isomorphism is given by $\xi \rightarrow \chi_{p}(\xi x)$, see e.g. 11, Section $2.3]$.

If $f \in L^{1}$ its Fourier transform is defined by

$$
(\mathcal{F} f)(\xi)=\int_{\mathbb{Q}_{p}} \chi_{p}(\xi x) f(x) d x, \quad \text { for } \xi \in \mathbb{Q}_{p}
$$

We will also use the notation $\mathcal{F}_{x \rightarrow \xi} f$ and $\widehat{f}$ for the Fourier transform of $f$. The Fourier transform is a linear isomorphism from $\mathcal{D}$ onto itself satisfying

$$
(\mathcal{F}(\mathcal{F} f))(\xi)=f(-\xi)
$$


for every $f \in \mathcal{D}$, see e.g. [1, Section 4.8]. If $f \in L^{2}$, its Fourier transform is defined as

$$
(\mathcal{F} f)(\xi)=\lim _{k \rightarrow \infty} \int_{|x|_{p} \leq p^{k}} \chi_{p}(\xi \cdot x) f(x) d^{n} x, \quad \text { for } \xi \in \mathbb{Q}_{p},
$$

where the limit is taken in $L^{2}$. We recall that the Fourier transform is unitary on $L^{2}$, i.e. $\|f\|_{L^{2}}=\|\mathcal{F} f\|_{L^{2}}$ for $f \in L^{2}$ and that (2.2) is also valid in $L^{2}$, see e.g. 21, Chapter III, Section 2].

2.4. The Vladimirov Operator. The Vladimirov pseudodifferential operator $\boldsymbol{D}^{\alpha}$, $\alpha>0$, is defined as

$$
\boldsymbol{D}^{\alpha} \varphi(x)=\frac{1-p^{\alpha}}{1-p^{-\alpha-1}} \int_{\mathbb{Q}_{p}}|y|_{p}^{-\alpha-1}(\varphi(x-y)-\varphi(x)) d y, \text { for } \varphi \in \mathcal{D} .
$$

The right-hand side of (2.3) makes sense for a wider class of functions, for example, for locally constant functions $\varphi$ satisfying

$$
\int_{|x|_{p} \geq 1}|x|_{p}^{-\alpha-d}|\varphi(x)| d x<\infty .
$$

Consequently, the constant functions are contained in the domain of $\boldsymbol{D}^{\alpha}$, and that $\boldsymbol{D}^{\alpha} \varphi=0$, for any constant function $\varphi$. On other hand,

$$
\boldsymbol{D}^{\alpha} \varphi(x)=\mathcal{F}_{\xi \rightarrow x}^{-1}\left(|\xi|_{p}^{\alpha} \mathcal{F}_{x \rightarrow \xi} \varphi\right), \text { for } \varphi \in \mathcal{D} .
$$

Finally in case in which the Vladimirov acts on functions depending on two variables, $(x, t) \in \mathbb{Q}_{p} \times \mathbb{R}_{+}$, we will sue the notation $\boldsymbol{D}_{x}^{\alpha} u(x, t)$ instead of $\boldsymbol{D}^{\alpha} u(x, t)$.

2.4.1. The spectrum of the operator $\boldsymbol{D}^{\alpha}$. The set of functions $\left\{\Psi_{r n j}\right\}$ defined as

$$
\Psi_{r n j}(x)=p^{\frac{-r}{2}} \chi_{p}\left(p^{-1} j\left(p^{r} x-n\right)\right) \Omega\left(\left|p^{r} x-n\right|_{p}\right),
$$

where $r \in \mathbb{Z}, j \in\{1, \cdots, p-1\}$, and $n$ runs through a fixed set of representatives of $\mathbb{Q}_{p} / \mathbb{Z}_{p}$, is an orthonormal basis of $L^{2}\left(\mathbb{Q}_{p}\right)$ consisting of eigenvectors of operator $D^{\alpha}$ :

$$
\boldsymbol{D}^{\alpha} \Psi_{r n j}=p^{(1-r) \alpha} \Psi_{r n j} \text { for any } r, n, j,
$$

We set $\mathcal{L}\left(B_{M}\right)$ to be the $\mathbb{C}$-vector space generated by the functions $\Psi_{r n j}(x)$ with support in $B_{M}$, which are exactly those satisfying

$$
r \leq M, n \in p^{r-M} \mathbb{Z}_{p} \cap \mathbb{Q}_{p} / \mathbb{Z}_{p}, \quad j \in\{1, \cdots, p-1\} .
$$

Notice that $\mathcal{L}\left(B_{M}\right)$ is a closed subspace of $L^{2}\left(B_{M}\right)$. Futhermore, all the functions $\Psi_{r n j}(x)$ satisfying (2.7) are orthogonal to the characteristic function of $B_{M}$, i.e. $\int_{B_{M}} \Psi_{r n j}(x) d x=0$. Notice that $L^{2}\left(B_{M}\right)=\mathbb{C} \Omega\left(p^{-M}|x|_{p}\right) \bigoplus L_{0}^{2}\left(B_{M}\right)$, where

$$
L_{0}^{2}\left(B_{M}\right)=\left\{f \in L^{2}\left(B_{M}\right) ; \int_{B_{M}} f d x=0\right\} .
$$


2.5. Two spectral problems. Consider the spectral problem:

$$
\left\{\begin{array}{l}
\boldsymbol{D}^{\alpha} \theta(x)=\kappa \theta(x), \quad \kappa \in \mathbb{R} \\
\theta \in L_{\mathbb{R}}^{2}\left(\mathbb{Q}_{p}\right) .
\end{array}\right.
$$

The functions $\Psi_{r n j}(x)$ are complex-valued eigenfunctions of (2.8) with eigenvalues $\kappa \in\left\{p^{(1-r) \alpha} ; r \in \mathbb{Z}\right\}$. Notice that each eigenvalues has infinite multiplicity. Therefore

$$
\begin{aligned}
& p^{\frac{-r}{2}} \cos \left(\left\{p^{-1} j\left(p^{r} x-n\right)\right\}_{p}\right) \Omega\left(\left|p^{r} x-n\right|_{p}\right), \\
& p^{\frac{-r}{2}} \sin \left(\left\{p^{-1} j\left(p^{r} x-n\right)\right\}_{p}\right) \Omega\left(\left|p^{r} x-n\right|_{p}\right),
\end{aligned}
$$

with $r, j, n$ as before, are real-valued eigenfunctions of (2.8) with $\kappa=p^{(1-r) \alpha}$. Notice that the eigenfunctions are not completely determined by the 'wavenumber' $\kappa$. The functions of the type (2.9) form a basis of $L_{\mathbb{R}}^{2}\left(\mathbb{Q}_{p}\right)$ (which is not necessarily orthonormal). More precisely, $f(x)=\sum_{r n j} A_{r n j} \Psi_{r n j}(x) \in L_{\mathbb{R}}^{2}\left(\mathbb{Q}_{p}\right)$ admits an expansion of the form

$$
\begin{aligned}
& \sum_{r n j} p^{\frac{-r}{2}} \operatorname{Re}\left(A_{r n j}\right) \cos \left(\left\{p^{-1} j\left(p^{r} x-n\right)\right\}_{p}\right) \Omega\left(\left|p^{r} x-n\right|_{p}\right)- \\
& \sum_{r n j} p^{\frac{-r}{2}} \operatorname{Im}\left(A_{r n j}\right) \sin \left(\left\{p^{-1} j\left(p^{r} x-n\right)\right\}_{p}\right) \Omega\left(\left|p^{r} x-n\right|_{p}\right),
\end{aligned}
$$

where

$$
\begin{aligned}
& \operatorname{Re}\left(A_{r n j}\right)=p^{\frac{-r}{2}} \int_{\mathbb{Q}_{p}} f(x) \cos \left(\left\{p^{-1} j\left(p^{r} x-n\right)\right\}_{p}\right) \Omega\left(\left|p^{r} x-n\right|_{p}\right) d x, \\
& \operatorname{Im}\left(A_{r n j}\right)=p^{\frac{-r}{2}} \int_{\mathbb{Q}_{p}} f(x) \sin \left(\left\{p^{-1} j\left(p^{r} x-n\right)\right\}_{p}\right) \Omega\left(\left|p^{r} x-n\right|_{p}\right) d x .
\end{aligned}
$$

Now we consider the eigenvalue problem:

$$
\begin{cases}\boldsymbol{D}_{x}^{\alpha} \theta(x)=\kappa \theta(x), & \kappa \in \mathbb{R} \\ \theta \in L_{\mathbb{R}}^{2}\left(B_{M}\right) \cap \mathcal{L}\left(B_{M}\right), & M \in \mathbb{Z} .\end{cases}
$$

All the functions $\operatorname{Re}\left(\Psi_{r n j}(x)\right)$ satisfying (2.7) are solutions of (2.11), with $\kappa=$ $p^{(1-r) \alpha}$. Now, any function $f \in L_{\mathbb{R}}^{2}\left(B_{M}\right) \cap \mathcal{L}\left(B_{M}\right)$ admits a Fourier expansion of the form

$$
f(x)=\sum_{r n j} C_{r n j} \Psi_{r n j}(x)=\sum_{r n j} \operatorname{Re}\left(C_{r n j} \Psi_{r n j}(x)\right),
$$

where the $\Psi_{r n j}(x)$ s run through all the wavelets supported in the ball $B_{M}$. Therefore, (2.12) is a solution of (2.11).

Finally we notice that

$$
\boldsymbol{D}^{\alpha} \Omega\left(p^{-M}|x|_{p}\right)=\left\{\begin{array}{lll}
\frac{\left(1-p^{-1}\right) p^{-\alpha M}}{1-p^{-\alpha-1}} & \text { if } & |x|_{p} \leq p^{M} \\
\frac{\left(1-p^{\alpha}\right) p^{M}}{1-p^{-\alpha-1}} \frac{1}{|x|_{p}^{\alpha+1}} & & |x|_{p}>p^{M}
\end{array}\right.
$$

implies that $\Omega\left(p^{-M}|x|_{p}\right)$ is not a solution of (2.11). 
2.6. The $p$-adic heat equation. The evolution equation

$$
\frac{\partial u(x, t)}{\partial t}+\left(\boldsymbol{D}_{x}^{\alpha} u\right)(x, t)=0, \quad x \in \mathbb{Q}_{p}, \quad t \geq 0,
$$

is the $p$-adic heat equation. The analogy with the classical heat equation comes from the fact that the solution of the initial value problem attached to (2.14) with initial datum $u(x, 0)=\varphi(x) \in \mathcal{D}_{\mathbb{R}}$ is given by

$$
u(x, t)=\int_{\mathbb{Q}_{p}} Z(x-y, t) \varphi(x) d x,
$$

where

$$
Z(x, t):=\int_{\mathbb{Q}_{p}} \chi_{p}(-x \xi) e^{-t|\xi|_{p}^{\alpha}} d \xi \text { for } t>0
$$

is the $p$-adic heat kernel. $Z(x, t)$ is a transition density of a time and space homogeneous Markov process which is bounded, right continuous and has no discontinuities other than jumps, see e.g. [30, Theorem 16].

We now review the classical case. For $\gamma>0$, consider the fractional Laplacian:

$$
(-\Delta)^{\gamma} \varphi(x)=\mathcal{F}_{\xi \rightarrow x}^{-1}\left(|\xi|_{\mathbb{R}}^{\gamma} \mathcal{F}_{x \rightarrow \xi} \varphi\right),
$$

where $\varphi$ is a Schwartz function, $\mathcal{F}$ denotes the Fourier transform in the group $(\mathbb{R},+)$. The heat kernel, as a distribution, is given by

$$
Z_{\mathbb{R}}(x, t)=\mathcal{F}_{\xi \rightarrow x}^{-1}\left(\exp -t|\xi|_{\mathbb{R}}^{\gamma}\right), \text { for } x \in \mathbb{R}, t>0 .
$$

In order to have a probabilistic meaning, this kernel must be a probability measure, then by a Bochner theorem, cf. [4, Theorem 3.12], exp $-t|\xi|_{\mathbb{R}}^{\gamma}$ is a positive definite function, and by a theorem due to Schoenberg cf. [4, Theorem 7.8], $|\xi|_{\mathbb{R}}^{\gamma}$ is a negative definite function. Now, if $\psi: \mathbb{R} \rightarrow \mathbb{C}$ is a negative definite function, then $|\psi(x)| \leq C|x|_{\mathbb{R}}^{2}$ for $|x|_{\mathbb{R}} \geq 1$, cf. [4, Corollary 7.16]. This implies that $0 \leq \gamma \leq 2$.

The family of ' $p$-adic Laplacians' is very large, see e.g. [11, Chapter 12], [10, Chapter 4], [24, Chapter 3, Section XVI] 30, Chapter 2] and the references therein.

\section{THE Model}

We fix $f, g: \mathbb{R}^{2} \rightarrow \mathbb{R}$ two $\mathbb{R}$-analytic functions, and fix $d, \gamma, \alpha>0$. In this article we consider the following non-Archimedean Turing system:

$$
\left\{\begin{array}{l}
u(\cdot, t), v(\cdot, t) \in L_{\mathbb{R}}^{2}\left(B_{M}\right) \cap \mathcal{L}\left(B_{M}\right), \text { for } t \geq 0 ; \\
u(x, 0), v(x, 0) \in L_{\mathbb{R}}^{2}\left(B_{M}\right) \cap \mathcal{L}\left(B_{M}\right), u(x, 0), v(x, 0) \geq 0 ; \\
\frac{\partial u}{\partial t}(x, t)=\gamma f(u, v)-\boldsymbol{D}_{x}^{\alpha} u(x, t) ; \\
\frac{\partial v}{\partial t}(x, t)=\gamma g(u, v)-d \boldsymbol{D}_{x}^{\alpha} v(x, t), x \in B_{M}, t \geq 0 .
\end{array}\right.
$$

Since we want to study self-organization patterns we need a 'zero flux boundary condition', which is

$$
u(x, t), v(x, t) \equiv 0 \text { for } x \in \mathbb{Q}_{p} \backslash B_{M}, \text { for any } t \geq 0 .
$$

Condition (3.2) seems very strong in comparison with the classical one, but there are at least to reasons for this choice. First, since $B_{M}$ is open and closed, then its boundary is the empty set. Second, the operator $\boldsymbol{D}_{x}^{\alpha}$ is non-local, as a consequence 
of this a condition like $\boldsymbol{D}_{x}^{\alpha} u(x, t) \equiv 0$ for any $x \in B_{M}$, for any $t \geq 0$, it is not sufficient to stop the diffusion outside of ball $B_{M}$, see (2.13).

\section{Turing Instability Criteria}

We now consider a homogeneous steady state $\left(u_{0}, v_{0}\right)$ of (3.1) which is a positive solution of

$$
f(u, v)=g(u, v)=0 .
$$

Since $u, v$ are real-valued functions, to study the linear stability of $\left(u_{0}, v_{0}\right)$ we can use the classical results, see e.g. [15, Chapter 2]. Following Turing, in the absence of any spatial variation, the homogeneous state must be linearly stable. With no spatial variation $u, v$ satisfy

$$
\left\{\begin{array}{c}
\frac{\partial u}{\partial t}(x, t)=\gamma f(u, v) \\
\frac{\partial v}{\partial t}(x, t)=\gamma g(u, v) .
\end{array}\right.
$$

Notice that (4.2) is an ordinary system of differential equations in $\mathbb{R}^{2}$. In order to linearize about the steady state $\left(u_{0}, v_{0}\right)$, we set

$$
\boldsymbol{w}=\left[\begin{array}{l}
w_{1} \\
w_{2}
\end{array}\right]=\left[\begin{array}{l}
u-u_{0} \\
v-v_{0} .
\end{array}\right]
$$

By using the fact that $f$ and $g$ are $\mathbb{R}$-analytic, and assuming that $\|\boldsymbol{w}\|_{L^{\infty}}:=$ $\max \left\{\left\|w_{1}\right\|_{L^{\infty}},\left\|w_{2}\right\|_{L^{\infty}}\right\}$ is small, then (4.2) can be approximated as

$$
\frac{\partial \boldsymbol{w}}{\partial t}=\gamma \rrbracket \boldsymbol{w},
$$

where

$$
\mathbb{J}=\left[\begin{array}{ll}
\frac{\partial f}{\partial u} & \frac{\partial f}{\partial v} \\
\frac{\partial g}{\partial u} & \frac{\partial g}{\partial v}
\end{array}\right]\left(u_{0}, v_{0}\right)=:\left[\begin{array}{cc}
f_{u_{0}} & f_{v_{0}} \\
g_{u_{0}} & g_{v_{0}}
\end{array}\right] .
$$

We now look for solutions of (4.4) of the form

$$
\boldsymbol{w}(t ; \lambda)=e^{\lambda t} \boldsymbol{w}_{0} .
$$

By substituting (4.5) in (4.4), the eigenvalues $\lambda$ are the solutions of

$$
\operatorname{det}(\gamma \mathbb{J}-\lambda I)=0,
$$

i.e.

$$
\lambda^{2}-\gamma(\operatorname{Tr} \mathbb{J}) \lambda+\gamma^{2} \operatorname{det} \mathbb{J}=0 .
$$

Consequently

$$
\lambda_{1,2}=\frac{\gamma}{2}\left\{\operatorname{Tr} \mathbb{I} \pm \sqrt{(\operatorname{Tr} \mathbb{J})^{2}-4 \operatorname{det} \mathbb{J}}\right\} .
$$

The steady state $\boldsymbol{w}=\mathbf{0}$ is linearly stable if $\operatorname{Re} \lambda_{1,2}<0$, this last condition is guaranteed if

$$
\operatorname{Tr} \mathbb{J}<0 \text { and } \operatorname{det} \mathbb{I}>0 \text {. }
$$


We linearize the full reaction-ultradiffusion system about the steady state, which is $\boldsymbol{w}=\mathbf{0}:=\left[\begin{array}{l}0 \\ 0\end{array}\right]$, see (4.3), to get

$$
\frac{\partial \boldsymbol{w}}{\partial t}(x, t)=\gamma \rrbracket \boldsymbol{w}(x, t)-D \boldsymbol{D}_{x}^{\alpha} \boldsymbol{w}(x, t),
$$

where

$$
D=\left[\begin{array}{ll}
1 & 0 \\
0 & d
\end{array}\right], \quad \boldsymbol{D}_{x}^{\alpha} \boldsymbol{w}:=\left[\begin{array}{c}
\boldsymbol{D}_{x}^{\alpha} \boldsymbol{w}_{1} \\
\boldsymbol{D}_{x}^{\alpha} \boldsymbol{w}_{2}
\end{array}\right] .
$$

To solve the system (4.8) subject to the boundary conditions (3.2), we first determine a solution $\boldsymbol{w}_{\kappa}$ of the following eigenvalue problem:

$$
\left\{\begin{array}{l}
\boldsymbol{D}_{x}^{\alpha} \boldsymbol{w}_{\kappa}(x)=\kappa \boldsymbol{w}_{\kappa}(x) \\
\boldsymbol{w}_{\kappa} \in L_{\mathbb{R}}^{2}\left(B_{M}\right) \cap \mathcal{L}\left(B_{M}\right) .
\end{array}\right.
$$

In Section 2.5, the existence of solution for the eigenvalue problem (4.10) was established. Indeed, if $\boldsymbol{w}_{\kappa}=\left[\begin{array}{c}w_{1, \kappa} \\ w_{2, \kappa}\end{array}\right]$, then

$$
\begin{gathered}
w_{1, \kappa}, w_{2, \kappa} \in \bigsqcup_{r n j}\left\{p^{\frac{-r}{2}} \cos \left(\left\{p^{-1} j\left(p^{r} x-n\right)\right\}_{p}\right) \Omega\left(\left|p^{r} x-n\right|_{p}\right)\right\} \sqcup \\
\bigsqcup_{r n j}\left\{p^{\frac{-r}{2}} \sin \left(\left\{p^{-1} j\left(p^{r} x-n\right)\right\}_{p}\right) \Omega\left(\left|p^{r} x-n\right|_{p}\right)\right\} \sqcup\left\{\Omega\left(p^{-M}|x|_{p}\right)\right\},
\end{gathered}
$$

with $r=r(\kappa), j \in\{1, \cdots, p-1\}$, and $n \in \mathbb{Q}_{p} / \mathbb{Z}_{p}$ as in (2.7). The value $\frac{p^{-\alpha M}}{1-p^{-\alpha-1}}$ is the eigenvalue corresponding to $\Omega\left(p^{-M}|x|_{p}\right)$. We now look for a solution $\boldsymbol{w}(x, t)$ of (4.8) of the form $\boldsymbol{w}(x, t)=\sum_{\kappa, \lambda} C_{\kappa, \lambda} e^{\lambda t} \boldsymbol{w}_{\kappa}(x)$. The function $e^{\lambda t} \boldsymbol{w}_{\kappa}(x)$ is a non-trivial solution of (4.8) if $\lambda$ satisfies

$$
\operatorname{det}(\lambda I-\gamma \mathbb{J}+\kappa D)=0,
$$

i.e.

$$
\lambda^{2}+\{\kappa(1+d)-\gamma \operatorname{Tr} \mathbb{J}\} \lambda+h(\kappa)=0,
$$

where

$$
h(\kappa):=d \kappa^{2}-\gamma \kappa\left(d f_{u_{0}}+g_{v_{0}}\right)+\gamma^{2} \operatorname{det} \mathbb{J} .
$$

In the Archimedean case, see e.g. 15, Section 2.3], condition (4.11) becomes condition (4.6) when $\kappa=0$. Since $\kappa=0$ is not an eigenvalue of operator $\boldsymbol{D}_{x}^{\alpha}$, conditions (4.11) and (4.6) are independent.

The steady state $\left(u_{0}, v_{0}\right)$ is linearly stable if both solutions of (4.12) have $\operatorname{Re}(\lambda)<$ 0 . Conditions (4.7) guarantee that the steady state is stable in absence of spatial effects, i.e. $\operatorname{Re}\left(\left.\lambda\right|_{\kappa=0}\right)<0$. For the steady state to be unstable to spatial disturbances we require $\operatorname{Re}(\lambda(\kappa))>0$ for some $\kappa \neq 0$. This can happen if either the coefficient of $\lambda$ in (4.12) is negative, or if $h(\kappa)<0$ for some $\kappa \neq 0$ in (4.13). Since $\operatorname{Tr} \mathbb{J}<0$ from conditions (4.7) and the coefficient of $\lambda$ in (4.13) is $(1+d)-\gamma \operatorname{Tr} \mathbb{J}$, which is positive, therefore, the only way $\operatorname{Re}(\lambda(\kappa))$ can be positive is if $h(\kappa)<0$ for some $\kappa \neq 0$. Since $\operatorname{det} \mathbb{J}>0$ from (4.7), in order $h(\kappa)$ to be negative, it is necessary that $\left(d f_{u_{0}}+g_{v_{0}}\right)>0$. Now, since $f_{u_{0}}+g_{v_{0}}=\operatorname{Tr} \mathbb{J}<0$, necessarily $d \neq 1$ 
and $f_{u_{0}}$ and $g_{v_{0}}$ must have opposite signs. So an additional requirement to those of (4.7) is

$$
d \neq 1 \text {. }
$$

This is a necessary condition, but not sufficient for $\operatorname{Re}(\lambda(\kappa))>0$. For $h(\kappa)$ to be negative for some nonzero $\kappa$, the minimum $h_{\min }$ of $h(\kappa)$ must be negative. An elementary calculation shows that

$$
h_{\min }=\gamma^{2}\left\{\operatorname{det} \mathbb{J}-\frac{\left(d f_{u_{0}}+g_{v_{0}}\right)^{2}}{4 d}\right\},
$$

and the minimum is achieved at

$$
\kappa_{\min }=\gamma \frac{d f_{u_{0}}+g_{v_{0}}}{2 d}
$$

Thus the condition $h(\kappa)<0$ for some $\kappa \neq 0$ is

$$
\frac{\left(d f_{u_{0}}+g_{v_{0}}\right)^{2}}{4 d}>\operatorname{det} \mathbb{J} .
$$

A bifurcation occurs when $h_{\min }=0$, see (4.15), for fixed kinetics parameters, this condition,

$$
\operatorname{det} \mathbb{J}=\frac{\left(d f_{u_{0}}+g_{v_{0}}\right)^{2}}{4 d},
$$

defines a critical diffusion $d_{c}$, which is given as an appropriate root of

$$
f_{u_{0}}^{2} d_{c}^{2}+2\left(2 f_{v_{0}} g_{u_{0}}-f_{u_{0}} g_{v_{0}}\right) d_{c}+g_{v_{0}}^{2}=0 .
$$

The model for $d>d_{c}$ exhibits Turing instability, while for $d<d_{c}$ no. Notice that $d_{c}>1$. A critical 'wavenumber' $\kappa_{c}$ is obtained by using (4.16):

$$
\kappa_{c}=\gamma \frac{d_{c} f_{u_{0}}+g_{v_{0}}}{2 d_{c}}=\gamma \sqrt{\frac{\operatorname{det} \mathbb{J}}{d_{c}}} .
$$

When $d>d_{c}$, there exists a range of unstable of positive wavenumbers $\kappa_{1}<\kappa<\kappa_{2}$, where $\kappa_{1}, \kappa_{2}$ are the zeros of $h(\kappa)=0$, see (4.13):

$$
\begin{aligned}
& \kappa_{1}=\frac{\gamma}{2 d}\left\{\left(d f_{u_{0}}+g_{v_{0}}\right)-\sqrt{\left(d f_{u_{0}}+g_{v_{0}}\right)^{2}-4 d \operatorname{det} \mathbb{J}}\right\}, \\
& \kappa_{2}=\frac{\gamma}{2 d}\left\{\left(d f_{u_{0}}+g_{v_{0}}\right)+\sqrt{\left(d f_{u_{0}}+g_{v_{0}}\right)^{2}-4 d \operatorname{det} \mathbb{J}}\right\} .
\end{aligned}
$$

We call the function $\lambda(\kappa)$ the dispersion relation. Notice that, within the unstable range, $\operatorname{Re} \lambda(\kappa)>0$ has a maximum for the wavenumber $\kappa_{\min }^{(0)}$ obtained from (4.16) with $d>d_{c}$. Some typical plots for $\lambda(\kappa)$ and $\operatorname{Re} \lambda(\kappa)$ are showed in [15, Section 2.3], see also the figure 2.5 in [15, Section 2.3]. Then as $t$ increases the behavior of $\boldsymbol{w}(x, t)$ is controlled by the dominant modes, i.e. those $e^{\lambda(\kappa) t} \boldsymbol{w}_{\kappa}(x)$ with $\operatorname{Re} \lambda(\kappa)>0$, since the other modes tend to zero exponentially. Then

$$
\begin{aligned}
\boldsymbol{w}(x, t) \sim & \sum_{\kappa_{1}<\kappa<\kappa_{2}} \sum_{n j} \boldsymbol{A}_{r n j} e^{\lambda t} p^{\frac{-r}{2}} \cos \left(\left\{p^{-1} j\left(p^{r} x-n\right)\right\}_{p}\right) \Omega\left(\left|p^{r} x-n\right|_{p}\right)+ \\
& \sum_{\kappa_{1}<\kappa<\kappa_{2}} \sum_{n j} \boldsymbol{B}_{r n j} e^{\lambda t} p^{\frac{-r}{2}} \sin \left(\left\{p^{-1} j\left(p^{r} x-n\right)\right\}_{p}\right) \Omega\left(\left|p^{r} x-n\right|_{p}\right)
\end{aligned}
$$


for $t \rightarrow+\infty$. In the above expansion in all the series the $r$ s and $j$ s take only a finite numbers of values. Indeed, all the $\kappa \mathrm{s}$ but one has the form $p^{(1-r) \alpha}$, the condition $\kappa_{1}<\kappa<\kappa_{2}$ implies that there is only a finite number of $r$ s. But the $n$ s run through an infinite set, see (2.7). We now fix $r$, then, for a given $x \in B_{M}$ there exists only a finite numbers of balls of type $B_{r}\left(p^{-r} n\right)$ containing $x$, see (2.7). This fact implies that the value at $(x, t)$ of $\boldsymbol{w}(x, t)$ in expansion (4.23) is determined only by a finite number of $n \mathrm{~s}$, and consequently the series in expansion (4.23) is convergent. We now formulate the Turing instability criteria for our model.

Notation 2. We denote by $\sigma\left(\boldsymbol{D}^{\alpha}\right)$ the spectra of $\boldsymbol{D}^{\alpha}$.

Theorem 1. Consider the reaction-diffusion system (3.1). The steady state $\left(u_{0}, v_{0}\right)$ is linearly unstable (Turing unstable) if the following conditions hold:

(T1) $\operatorname{Tr} A=f_{u_{0}}+g_{v_{0}}<0$;

(T2) $\operatorname{det} \mathbb{A}=f_{u_{0}} g_{v_{0}}-f_{v_{0}} g_{u_{0}}>0$;

(T3) $d f_{u_{0}}+g_{v_{0}}>0$

(T4) the derivatives $f_{u_{0}}$ and $g_{v_{0}}$ must have opposite signs;

(T5) $\left(d f_{u_{0}}+g_{v_{0}}\right)^{2}-4 d\left(f_{u_{0}} g_{v_{0}}-f_{v_{0}} g_{u_{0}}\right)>0$;

(T6) $\left\{\kappa \in \sigma\left(\boldsymbol{D}^{\alpha}\right) ; \kappa_{1}<\kappa<\kappa_{2}\right\} \neq \emptyset$.

Moreover, there are infinitely many unstable eigenmodes, and the Turing pattern has the form 4.23).

\section{Discrete Models of Morphogenesis}

5.1. The spaces $\mathcal{D}_{M}^{-L}$. We fix $M \in \mathbb{Z}$ and $L \in \mathbb{N}$, with $L \geq-M$, and define

$$
G_{L, M}=p^{-M} \mathbb{Z}_{p} / p^{L} \mathbb{Z}_{p}
$$

Then, $G_{L, M}$ is a finite ring, with $\# G_{L, M}=p^{L+M}$ elements. We fix the following a set of representatives for $G_{L, M}$ :

$$
I=I_{-M} p^{-M}+I_{-M+1} p^{-M+1}+\ldots+I_{L-1} p^{L-1},
$$

where the $I_{j}$ s are $p$-adic digits, i.e. elements from $\{0,1, \ldots, p-1\}$.

On the other hand, since $I=p^{-M} \widetilde{I}$, with

$$
\widetilde{I}=I_{-M}+I_{-M+1} p+\ldots+I_{L-1} p^{L+M-1} \in \mathbb{Z}_{p} / p^{L+M} \mathbb{Z}_{p}
$$

and elements of $\mathbb{Z}_{p} / p^{L+M} \mathbb{Z}_{p}$ form in a natural way a rooted tree with $L+M$ levels, where the level $1 \leq j \leq L+M$ is formed by the elements of the form $a_{0}+\ldots+a_{j-1} p^{j-1}$. In this way we can identify $G_{L, M}$ with a rooted tree. This a identification is not unique, see e.g. [29].

By considering $G_{L, M}$ as a subset of $\mathbb{Q}_{p}$, we can endow $G_{L, M}$ with a norm denoted as $|\cdot|_{p}$, and thus $\left(G_{L, M},|\cdot|_{p}\right)$ becomes a finite ultrametric space.

We define $\mathcal{D}_{M}^{-L}$ to be the $\mathbb{R}$-vector space formed by test functions $\varphi$ supported in the ball $p^{-M} \mathbb{Z}_{p}$ having the form

$$
\varphi(x)=p^{\frac{L}{2}} \sum_{I \in G_{L, M}} \varphi(I) \Omega\left(p^{L}|x-I|_{p}\right), \text { with } \varphi(I) \in \mathbb{R} .
$$

Since $\Omega\left(p^{L}|x-I|_{p}\right) \Omega\left(p^{L}|x-J|_{p}\right)=0$ if $I \neq J$, the set

$$
\left\{p^{\frac{L}{2}} \Omega\left(p^{L}|x-I|_{p}\right) ; I \in G_{L, M}\right\}
$$


is an orthonormal basis for $\mathcal{D}_{M}^{-L}$. Now, by using that

$$
\begin{aligned}
\|\varphi\|_{L^{2}} & =\sqrt{p^{L} \sum_{I \in G_{L, M}}|\varphi(I)|^{2} \int_{p^{-M} \mathbb{Z}_{p}} \Omega\left(p^{L}|x-I|_{p}\right) d x} \\
& =\sqrt{\sum_{I \in G_{L, M}}|\varphi(I)|^{2}}
\end{aligned}
$$

we have

$$
\left(\mathcal{D}_{M}^{-L},\|\cdot\|_{L^{2}}\right) \simeq\left(\mathbb{R}^{\# G_{L, M}},\|\cdot\|_{\mathbb{R}}\right) \text { as Banach spaces, }
$$

where $\|\cdot\|_{\mathbb{R}}$ denotes the usual norm of $\mathbb{R}^{\# G_{L, M}}$.

5.2. Discretization of the operator $\boldsymbol{D}^{\alpha}$. We set $\lambda_{M}:=\frac{\left(1-p^{-1}\right) p^{-\alpha M}}{1-p^{-\alpha-1}}>0$. Notice that

$$
\boldsymbol{D}_{x}^{\alpha} \varphi(x)=\left(\boldsymbol{D}_{M}^{\alpha}-\lambda_{M}\right) \varphi(x) \text { for } \varphi \in \mathcal{D}_{\mathbb{R}}\left(p^{-M} \mathbb{Z}_{p}\right),
$$

where

$$
\boldsymbol{D}_{M}^{\alpha} \varphi(x):=\frac{1-p^{\alpha}}{1-p^{-\alpha-1}} \int_{p^{-M} \mathbb{Z}_{p}} \frac{\varphi(x-y)-\varphi(x)}{|y|_{p}^{\alpha+1}} d y .
$$

The operator $\boldsymbol{D}_{M}^{\alpha}-\lambda_{M}$ is a non-negative, symmetric operator on $L_{\mathbb{R}}^{2}\left(p^{-M} \mathbb{Z}_{p}\right)$. Furthermore, its closure, also denoted by $\boldsymbol{D}_{M}^{\alpha},-\lambda_{M}$ is a self-adjoint operator, see [10, Section 3.3.2].

Every wavelet $\Psi_{r n j}(x)$, with support in $p^{-M} \mathbb{Z}_{p}$, with $r, n, j$ satisfying (2.7), is an eigenfunction of $\boldsymbol{D}_{M}^{\alpha}$ with eigenvalue $p^{(1-r) \alpha}$. In addition $p^{\frac{M}{2}} \Omega\left(p^{-M}|x|_{p}\right)$ is also a eigenfunction of $\boldsymbol{D}_{M}^{\alpha}$ with eigenvalue $\lambda_{M} p^{\frac{M}{2}}$.

As a discretization of $\boldsymbol{D}_{M}^{\alpha}-\lambda_{M}$, we pick its restriction to $\mathcal{D}_{M}^{-L}$, which is denoted as $\boldsymbol{D}_{L, M}^{\alpha}-\lambda_{M}$. Since $\mathcal{D}_{M}^{-L}$ is a finite vector space $\boldsymbol{D}_{L, M}^{\alpha}-\lambda_{M}$ is represented by a matrix $A_{L, M}^{\alpha}$.

5.3. Computation of the matrix $A_{L, M}^{\alpha}$. In order to compute the matrix $A_{L, M}^{\alpha}$, we first compute

$$
\begin{aligned}
& \boldsymbol{D}_{M}^{\alpha}\left(p^{\frac{L}{2}} \Omega\left(p^{L}|x-I|_{p}\right)\right) \\
& =p^{\frac{L}{2}} \frac{1-p^{\alpha}}{1-p^{-\alpha-1}} \int_{p^{-M} \mathbb{Z}_{p}} \frac{\Omega\left(p^{L}|x-y-I|_{p}\right)-\Omega\left(p^{L}|x-I|_{p}\right)}{|y|_{p}^{\alpha+1}} d y \\
& =p^{\frac{L}{2}} \frac{1-p^{\alpha}}{1-p^{-\alpha-1}} \sum_{J \in G_{L, M}} \int_{|y-J|_{p} \leq p^{-L}} \frac{\Omega\left(p^{L}|x-y-I|_{p}\right)-\Omega\left(p^{L}|x-I|_{p}\right)}{|y|_{p}^{\alpha+1}} d y \\
& =: p^{\frac{L}{2}} \frac{1-p^{\alpha}}{1-p^{-\alpha-1}} \sum_{J \in G_{L, M}} \mathcal{I}_{J}(x, I, L) .
\end{aligned}
$$

We now compute the integrals $\mathcal{I}_{J}(x, I, L)$. We consider first the case $J \neq 0$. By using that

$$
\Omega\left(p^{L}|x-I|_{p}\right) * \Omega\left(p^{L}|x-J|_{p}\right)=p^{-L} \Omega\left(p^{L}|x-(I+J)|_{p}\right),
$$


we have

$$
\begin{aligned}
\mathcal{I}_{J}(x, I, L) & =\frac{1}{|J|_{p}^{\alpha+1}}\left\{\Omega\left(p^{L}|x-I|_{p}\right) * \Omega\left(p^{L}|x-J|_{p}\right)-p^{-L} \Omega\left(p^{L}|x-I|_{p}\right)\right\} \\
& =\frac{p^{-L}}{|J|_{p}^{\alpha+1}}\left\{\Omega\left(p^{L}|x-(I+J)|_{p}\right)-\Omega\left(p^{L}|x-I|_{p}\right)\right\} .
\end{aligned}
$$

Now in the case $J=0$, we have $\mathcal{I}_{J}(x, I, L)=0$. Indeed,

$$
\mathcal{I}_{0}(x, I, L)=\int_{|y|_{p} \leq p^{-L}} \frac{\Omega\left(p^{L}|x-y-I|_{p}\right)-\Omega\left(p^{L}|x-I|_{p}\right)}{|y|_{p}^{\alpha+1}} d y .
$$

If $\Omega\left(p^{L}|x-I|_{p}\right)=1$, then $x \in I+p^{L} \mathbb{Z}_{p}$ and since $y \in p^{L} \mathbb{Z}_{p}$ we have $x-y-I \in$ $p^{L} \mathbb{Z}_{p}$, which implies that $\Omega\left(p^{L}|x-y-I|_{p}\right)=1$. Now, if $\Omega\left(p^{L}|x-I|_{p}\right)=0$, i.e. if $x \in K+p^{L} \mathbb{Z}_{p}$ for some $K \neq I$, then by using that $p^{L} \mathbb{Z}_{p} \cap K-I+p^{L} \mathbb{Z}_{p}=\varnothing$, we have

$$
\mathcal{I}_{0}(x, I, L)=\int_{p^{L} \mathbb{Z}_{p} \cap K-I+p^{L} \mathbb{Z}_{p}} \frac{1}{|y|_{p}^{\alpha+1}} d y=0 .
$$

Now, we use the fact that $G_{L, M}$ is a additive group to conclude that

$$
\begin{gathered}
\boldsymbol{D}_{M}^{\alpha}\left(p^{\frac{L}{2}} \Omega\left(p^{L}|x-I|_{p}\right)\right) \\
=p^{-\frac{L}{2}} \frac{1-p^{\alpha}}{1-p^{-\alpha-1}} \sum_{\substack{K \in G_{L, M} \\
K \neq I}} \frac{1}{|K-I|_{p}^{\alpha+1}} \Omega\left(p^{L}|x-K|_{p}\right) \\
-p^{-\frac{L}{2}} \frac{1-p^{\alpha}}{1-p^{-\alpha-1}}\left(\sum_{\substack{K \in G_{L, M} \\
K \neq I}} \frac{1}{|K-I|_{p}^{\alpha+1}}\right) \Omega\left(p^{L}|x-I|_{p}\right),
\end{gathered}
$$

and the entries of the matrix $A_{L, M}^{\alpha}=\left[A_{K, I}^{\alpha}\right]_{K, I \in G_{L, M}}$ are given as

$$
A_{K, I}^{\alpha}=\left\{\begin{array}{cc}
p^{-\frac{L}{2}} \frac{1-p^{\alpha}}{1-p^{-\alpha-1}} \frac{1}{|K-I|_{p}^{\alpha+1}} \quad \text { if } \quad K \neq I \\
-p^{-\frac{L}{2}} \frac{1-p^{\alpha}}{1-p^{-\alpha-1}} \sum_{K \neq I} \frac{1}{|K-I|_{p}^{\alpha+1}}-\lambda_{M} & \text { if } \quad K=I .
\end{array}\right.
$$

5.4. Discretization of the $p$-adic Turing System. In the discretization of the Turing system (3.1), we use the following approximation for functions $u(x, t)$, $v(x, t)$ :

$$
u^{(L)}(x, t)=\sum_{I \in G_{L, M}} u^{(L)}(I, t) \Omega\left(p^{L}|x-I|_{p}\right)
$$

and

$$
v^{(L)}(x, t)=\sum_{I \in G_{L, M}} v^{(L)}(I, t) \Omega\left(p^{L}|x-I|_{p}\right),
$$


where $u^{(L)}(I, \cdot), v^{(L)}(I, \cdot) \in C^{1}([0, T])$ for some fixed positive $T$. Furthermore, we set

$$
u^{(L)}(x, t)=\left[u^{(L)}(I, t)\right]_{I \in G_{L, M}}, v^{(L)}(x, t)=\left[v^{(L)}(I, t)\right]_{I \in G_{L, M}} .
$$

We assume that range $\left(u^{(L)}(x, t)\right) \times$ range $\left(v^{(L)}(x, t)\right)$ is contained in the domains of convergence of $f, g$. Then

$$
\begin{gathered}
f\left(\sum_{I \in G_{L, M}} u^{(L)}(I, t) \Omega\left(p^{L}|x-I|_{p}\right), \sum_{J \in G_{L, M}} v^{(L)}(J, t) \Omega\left(p^{L}|x-J|_{p}\right)\right)= \\
\sum_{I \in G_{L, M}} f\left(u^{(L)}(I, t), v^{(L)}(I, t)\right) \Omega\left(p^{L}|x-I|_{p}\right) .
\end{gathered}
$$

A similar formula holds for function $g$. Then the discretization of the $p$-adic Turing system has the form:

$$
\begin{gathered}
\frac{\partial}{\partial t}\left[u^{(L)}(I, t)\right]_{I \in G_{L, M}}=\gamma\left[f\left(u^{(L)}(I, t), v^{(L)}(I, t)\right)\right]_{I \in G_{L, M}} \\
-A_{L, M}^{\alpha}\left[u^{(L)}(I, t)\right]_{I \in G_{L, M}} \\
\begin{aligned}
\frac{\partial}{\partial t}\left[v^{(L)}(I, t)\right]_{I \in G_{L, M}}=\gamma\left[g\left(u^{(L)}(I, t), v^{(L)}(I, t)\right)\right]_{I \in G_{L, M}} \\
-d A_{L, M}^{\alpha}\left[v^{(L)}(I, t)\right]_{I \in G_{L, M}}
\end{aligned}
\end{gathered}
$$

where $I \in G_{L, M}$. Equivalently,

$$
\begin{gathered}
\frac{\partial}{\partial t} u^{(L)}(I, t)=\gamma f\left(u^{(L)}(I, t), v^{(L)}(I, t)\right)-p^{-\frac{L}{2}} \frac{1-p^{\alpha}}{1-p^{-\alpha-1}} \sum_{J \neq I} \frac{u^{(L)}(J, t)}{|J-I|_{p}^{\alpha+1}} \\
+p^{-\frac{L}{2}} \frac{1-p^{\alpha}}{1-p^{-\alpha-1}}\left(\sum_{J \neq I} \frac{1}{|J-I|_{p}^{\alpha+1}}-\frac{\left(1-p^{-1}\right) p^{-\alpha M+\frac{L}{2}}}{1-p^{-\alpha}}\right) u^{(L)}(I, t), \\
\frac{\partial}{\partial t} v^{(L)}(I, t)=\gamma g\left(u^{(L)}(I, t), v^{(L)}(I, t)\right)-d p^{-\frac{L}{2}} \frac{1-p^{\alpha}}{1-p^{-\alpha-1}} \sum_{J \neq I} \frac{u^{(L)}(J, t)}{|J-I|_{p}^{\alpha+1}} \\
+d p^{-\frac{L}{2}} \frac{1-p^{\alpha}}{1-p^{-\alpha-1}}\left(\sum_{J \neq I} \frac{1}{|J-I|_{p}^{\alpha+1}}-\frac{\left(1-p^{-1}\right) p^{-\alpha M+\frac{L}{2}}}{1-p^{-\alpha}}\right) u^{(L)}(I, t),
\end{gathered}
$$

where $I \in G_{L, M}$.

\section{REFERENCES}

[1] S. Albeverio, A. Yu. Khrennikov, V. M. Shelkovich, Theory of p-adic distributions: linear and nonlinear models, London Mathematical Society Lecture Note Series, 370 (Cambridge University Press, 2010).

[2] B. Ambrosio, M. A. Aziz-Alaoui, V. L. E. Phan, Global attractor of complex networks of reaction-diffusion systems of Fitzhugh-Nagumo type, Discrete Contin. Dyn. Syst., Ser. B 23 (2018), No. 9, 3787-3797.

[3] Barrat, A., Barthélemy, M. \& Vespignani, A. Dynamical Processes on Complex Networks (Cambridge Univ. Press, 2008). 
[4] Berg Christian, Forst Gunnar, Potential theory on locally compact abelian groups (SpringerVerlag, New York-Heidelberg, 1975).

[5] S. Boccaletti, V. Latora, Y. Moreno, M. Chavez, D.-U. Hwang, Complex networks: structure and dynamics, Phys. Rep. 424 (2006), no. 4-5, 175-308.

[6] Soon-Yeong Chung, Jae-Hwang Lee, Blow-up for discrete reaction-diffusion equations on networks, Appl. Anal. Discrete Math. 9 (2015), No. 1, 103-119.

[7] Paul R. Halmos, Measure Theor (D. Van Nostrand Company, 1950).

[8] Horsthemke, W., Lam, K. \& Moore, P. K. Network topology and Turing instability in small arrays of diffusively coupled reactors, Phys. Lett. A 328, 444-451 (2004)

[9] Yusuke Ide, Hirofumi Izuhara, Takuya Machida, Turing instability in reaction-diffusion models on complex networks, Physica A 457 (2016), 331-347.

[10] Anatoly N. Kochubei, Pseudo-differential equations and stochastics over non-Archimedean fields (Marcel Dekker, 2001).

[11] Andrei Khrennikov, Sergei Kozyrev, W. A. Zúñiga-Galindo, Ultrametric Equations and its Applications, Encyclopedia of Mathematics and its Applications (168) (Cambridge University Press, 2018).

[12] M. Mocarlo Zheng, Bin Shao, Qi Ouyang, Identifying network topologies that can generate Turing pattern, J. Theor. Biol. 408 (2016), 88-96.

[13] Moore, P. K. \& Horsthemke, W. Localized patterns in homogeneous networks of diffusively coupled reactors, Physica D 206 (2005), 121-144.

[14] Delio Mugnolo, Semigroup methods for evolution equations on networks. Understanding Complex Systems (Springer, Cham, 2014).

[15] J. D. Murra, Mathematical biology. II. Spatial models and biomedical applications. Third edition (Springer-Verlag, New York, 2003)

[16] Hiroya Nakao, Alexander S. Mikhailov, Turing patterns in network-organized activator inhibitor systems, Nature Physics 6 (2010), 544-550.

[17] H. G. Othmer, L. E. Scriven, Instability and dynamic pattern in cellular networks, J. Theor. Biol. 32 (1971), 507-537.

[18] H. G. Othmer, L. E. Scriven, Nonlinear aspects of dynamic pattern in cellular networks, J. Theor. Biol. 43 (1974), 83-112.

[19] Benoît Perthame, Parabolic equations in biology. Growth, reaction, movement and diffusion, Lecture Notes on Mathematical Modelling in the Life Sciences (Springer, Cham, 2015).

[20] Angela Slavova, Pietro Zecca, Complex behavior of polynomial FitzHugh-Nagumo cellular neural network model, Nonlinear Anal., Real World Appl. 8 (2007), No. 4, 1331-1340.

[21] M. H. Taibleson, Fourier analysis on local fields (Princeton University Press, 1975).

[22] A. M. Turing, The chemical basis of morphogenesis, Philos. Trans. Roy. Soc. London Ser. B 237 (1952), no. 641, 37-72.

[23] Piet Van Mieghem, Graph spectra for complex networks (Cambridge University Press, Cambridge, 2011).

[24] V. S. Vladimirov, I. V. Volovich, E. I. Zelenov, p-adic analysis and mathematical physics (World Scientific, 1994)

[25] Joachim von Below, José A. Lubary, Instability of stationary solutions of reaction-diffusionequations on graphs, Result. Math. 68 (2015), No. 1-2, 171-201.

[26] Hongyong Zhao, Xuanxuan Huang, Xuebing Zhang, Turing instability and pattern formation of neural networks with reaction-diffusion terms, Nonlinear Dyn. 76 (2014), No. 1, 115-124.

[27] YusukeIde, Hirofumi Izuhara, Takuya Machida, Turing instability in reaction-diffusion models on complex networks, Phys. A 457 (2016), 331-347.

[28] W. A. Zúñiga-Galindo, Reaction-diffusion equations on complex networks and Turing patterns, via p-adic analysis, J. Math. Anal. Appl. 491 (2020), no. 1, 124239, 39 pp.

[29] W. A. Zúñiga-Galindo, Non-Archimedean Reaction-Ultradiffusion Equations and Complex Hierarchic Systems, Nonlinearity 31 (2018), no. 6, 2590-2616.

[30] W. A. Zúñiga-Galindo, Pseudodifferential equations over non-Archimedean spaces, Lectures Notes in Mathematics 2174 (Springer, Cham, 2016). 
University of Texas Rio Grande Valley, School of Mathematical \& Statistical Sciences, One West University Blyd, Brownsville, tX 78520, United States and Centro de Investigación y de Estudios Avanzados del Instituto Politécnico Nacional, Departamento de Matemáticas, Unidad Querétaro, Libramiento Norponiente \#2000, Fracc. Real de Juriquilla. Santiago de Querétaro, Qro. 76230, México.

Email address: wilson.zunigagalindo@utrgv.edu, wazuniga@math.cinvestav.edu.mx 